\title{
Neonatal Marfan Syndrome
}

\author{
Eleonora Tognato, $\mathrm{MD}^{1}$ Anna Perona, MD ${ }^{1}$ Angela Aronica, $\mathrm{MD}^{1}$ Antonella Bertola, MD ${ }^{1}$ \\ Lina Cimminelli, MD ${ }^{1}$ Simona De Vecchi, MD ${ }^{2} \quad$ Mohammad Reza Eshraghy, MD ${ }^{1} \quad$ Bruna Loperfido, MD ${ }^{1}$ \\ Claudia Vivenza, $\mathrm{MD}^{1}$ Paolo Manzoni, $\mathrm{MD}^{1}$
}

1 Division of Pediatrics and Neonatology, Department of Maternal and
Infant Medicine, Degli Infermi Hospital, Biella, Italy
${ }^{2}$ Division of Cardiology, Department of Medicine and Urgency, Degli
Infermi Hospital, Biella, Italy

Am J Perinatol 2019;36(suppl S2):S74-S76.
Address for correspondence Eleonora Tognato, MD, Division of Pediatrics, Neonatal and Infant Medicine, Department of Maternal Medicine, Nuovo Ospedale degli Infermi, ASL Biella, Via dei Ponderanesi, 2-13058, Ponderano, Biella, Italy (e-mail: eleonora.tognato@aslbi.piemonte.it).

\author{
Abstract \\ Keywords \\ - neonatal Marfan \\ syndrome \\ - Ghent criteria \\ - severe cardiovascular \\ disease \\ - neonate
}

Objective The Marfan syndrome (MFS) is an autosomal dominant disorder of connective tissue resulting from pathogenic variants of the fibrillin-1 gene (FBN1) with skeletal, cardiac, and ocular involvement.

Study Design We report on a full-term male neonate, who showed at birth characteristics and dysmorphisms suggestive of nMFS, combined with the detection of severe cardiovascular disease. A multidisciplinary team made up of neonatologists and pediatricians, cardiologists, geneticists, ophtalmologists, physiatrists and physioterapists was formed to manage this patient.

Results and Conclusion Early diagnosis of this rare condition is critical for adequate treatment and specific follow-up, and impacts significantly on prognosis.
The Marfan syndrome (MFS) is an autosomal dominant disorder of connective tissue resulting from pathogenic variants of the fibrillin-1 gene (FBN1) with skeletal, cardiac, and ocular involvement. Neonatal Marfan syndrome $(\mathrm{nMFS})^{1-3}$ is rare and it is the most severe form of this disease. NMFS has a poor prognosis, with a mean survival age of only 16.3 months. ${ }^{4}$ The main reason for death is congestive heart failure (CHF). ${ }^{5}$ Early diagnosis of nMFS allows a timely approach and an appropriate, multidisciplinary follow-up, making, thus, possible to identify early complications with subsequent improvement of an otherwise poor prognosis, since nMFS carries a high likelihood to cause mortality in infancy or early childhood.

\section{Materials and Methods}

We report on a full-term male neonate, born from unplanned Cesarean section due to failure of progression during labor. His birth weight was 3,690 g (70th centile), length $56 \mathrm{~cm}$ (100th centile), and head circumference $36 \mathrm{~cm}$ (86th centile). His Apgar's score was 7 and 9 at 1 st and 5 th minutes, respectively. There was no family history of inherited disorders and/or congenital heart disease. On 34th and 39th weeks of preg- nancy, ultrasonography reported a femoral length higher than 95th centile as well as oligohydramnios. At birth, the neonate featured several dysmorphisms, namely enophthalmos, dawnslanting palpebral fissure, crumpled ears, retromicrognathia, with characteristic "senile" facial appearance, redundant skin folds, wrist and thumb sign, dolichostenomelia, limited extension of the elbow and knee joint, long slenders fingers, and toes with arachnodactyly, pectus carinatum, dorsolumbar scoliosis, and plain pes planus. Cardiac examination revealed a grade 2/6 systolic murmur. Echocardiography showed ectasia aortic root at sinuses of the Valsalva $(Z$-score $>2)$, proleptic mitral valve with severe regurgitation $(2+/ 3+)$, and proleptic tricuspid valve with low to moderate regurgitation. Patent ductus arteriosus (spontaneously closing at 11 days of life) and foramen ovale pervium with minor left-right shunt were also detected; echocardiogram (ECG) tracing was normal. Ophthalmological examination revealed lens subdislocation. The chest and abdomen X-ray, the ultrasound study of his abdomen, and brain were normal. The evoked auditory potential test gave normal results. Chromosomal investigation showed a 46XY pattern. According to the revised Ghent criteria for the diagnosis of MFS, ${ }^{6}$ the above-listed series of dysmorphisms (score $>7$ ) combined with the detection of severe cardiovascular
Copyright (c) 2019 by Thieme Medical Publishers, Inc., 333 Seventh Avenue, New York, NY 10001, USA. Tel: +1(212) 584-4662
Dol https://doi.org/ 10.1055/s-0039-1691770. ISSN 0735-1631. 
disease prompted ultimately to the diagnosis of nMFS. Genetic investigations through molecular and sequencing techniques showed a mutation in heterozygosis on the fibrillin 1 (FBN1) gene: c.3143T > C (p.Ile1048Thr) with amino acid substitution of an isoleucine by a tyrosine at position 1,048 .

\section{Outcome and Follow-up}

The neonate showed early respiratory distress requiring supplemental oxygen in the first few days of life. He was given antacid medications until the 3rd month of life due to severe gastroesophageal reflux. At 3 months of age, he underwent surgery for left inguinal herniation. At 1 month of age, ECG revealed a moderate worsening of the aortic root's dilatation and mitral valve's prolapse, hence diuretic therapy with furosemide was initiated and maintained over time. At 8 months of age, due to the progressive worsening of fatigue and dyspnea with failure to thrive, he added treatment with enalapril. Nevertheless, there was a progressive aggravation of the mitral and tricuspid valve's prolapse and regurgitation with great dilation of the right atrium (area $20 \mathrm{~cm}^{2}$ ), left atrium (area $8 \mathrm{~cm}^{2}$ ), and aortic root $(30 \mathrm{~mm}$ ) that conditioned cardiothoracic surgery (mitral and tricuspidal valvuloplasty) at 11 months of age. He was discharged 9 days after surgery on furosemide, captopril, carvedilol, and omeprazole. He is currently followed-up by a multidisciplinary team including pediatricians, cardiologists, ophthalmologists, physiotherapists, and rehabilitation nurses, along with geneticists. Currently, he is 13 months old, clinically stable, with a good catch-up growth after heart surgery (weight 7,820 kg, 3rd centile; length $80 \mathrm{~cm}$, 80th centile; and head circumference $47 \mathrm{~cm}$, 50th centile). He has an adequate neuropsychomotor development for his age.

\section{Discussion}

The MFS is a heritable disorder of connective tissue resulting from pathogenic variants of the FBN1, a large gene composed of 65 exons on chromosome 15q21.1. The gene encoder FBN1, a $320 \mathrm{kDa}$ glycoprotein, is a main component of microfibrils in the extracellular matrix. The three foremost systems affected by this condition are the cardiovascular, ocular, and skeletal; being cardinal manifestation, (a) the aortic aneurysm with dissection, (b) ectopia lentis, and (c) long-bone overgrowth. This syndrome is notable for its variable expression, namely, in the severity of clinical manifestations, age at onset, and targeted tissues, among and within affected families. It can range from the most sever phenotype manifested in the neonatal period (nMFS) to a very mild clinical form. Owing to the penetrance of some features that is age-dependent, the Ghent criteria must be used with caution in children. ${ }^{1}$ An international expert panel has established a revised Ghent nosology, which emphasizes the cardiovascular manifestations and identifies the aortic root aneurysm and the ectopia lentis as the cardinal clinical features. In the absence of any family history, the presence of these two manifestations is sufficient for the unequivocal diagnosis of MFS. In absence of either of these two, the presence of a bona fide FBN1 mutation or a combination of systemic manifestations is required. As for the latter, a new scoring system has been designed. In this revised nosology, FBN1 testing, although not mandatory, has greater weight in the diagnostic assessment. ${ }^{2}$

The nMFS is a rare condition relative to classic and incomplete MFS, and has the most severe phenotype and the worst prognosis. Its incidence is far lower than that estimated for MFS, that is, $1 / 5,000$ to $1 / 10,000 .^{7}$ There are both genotype and phenotype differences between nMFS and classic and incomplete MFS. Based on information from the Universal Mutation Database-FBN1 mutations database, some $92 \%$ of nMFS mutations were de novo, which is significantly higher than the number of de novo classic and incomplete MFS mutations (35.3\%). Family history is negative in 70 to $100 \%$ of nMFS; however, whereas it is negative in only 20 to $30 \%$ of classic MFS patiens. ${ }^{5}$ As for the genome, the distribution of the two types of mutations differs among FBN1 exons; in particular, most nMFS mutations (86.4\%) cluster within exons 24 to 33, while the distribution of mutations for classic and incomplete MFS is more even with only $17.4 \%$ in the exon 24 to 33 region. Some $91.5 \%$ of nMFS mutations are located in cbEGF domains of which 43 (91.5\%) affect the disulfide bond or $\mathrm{Ca}^{2+}$ binding site. An nMFS genotype-phenotype analysis showed that most of the mutations (88.4\%) present exclusively in patients with nMFS. These observations strongly suggest that limited phenotype heterogeneity of nMFS-associated mutations is evident, although it should not be ignored that some mutations can also result in a later onset or classic presentation of MFS. ${ }^{3}$ The nMFS may be manifested in the prenatal period and is characterized, at birth, by some other features beyond the ones it shares with the classic form. These include flexion contractures, characteristic facial dysmorphology (crumpled ears, loose redundant skin, and a characteristic "senile" facial appearance), pulmonary emphysema, and severe cardiovascular disease. Mutations in the so-called "neonatal region" of FBN1, exons 24 to 33 are associated with a high risk of rapidly worsening cardiac disease. In contrast to the classic syndrome, in which main cause of death is aortic dissection or rupture, nMFS patients die mostly from CHF associated with mitral and tricuspid regurgitations and dilated aortic root. Skeletal manifestation, such as arachnodactyly, dolichostenomelia, and pectus deformities are typically present. ${ }^{2,5}$ Medical therapies are not very successful in nMFS as CHF is often not responsive to medications, and valvular regurgitations are progressing severely and rapidly. Surgery may be needed to repair or replace the mitral or tricuspid valves and to replace the aortic root if this last is involved. However, surgery is difficult in infants due to the high risk for mortality and morbidity including complete heart block, thrombosis, and stroke. Heart transplantation may be the last option for patients with nMFS. ${ }^{8}$ Early diagnosis of this rare condition is critical for adequate management and treatment, with a clear impact on the prognosis. A constant update on the knowledge and information about the nMFS mutation spectrum is helpful to improve our understanding of genotypephenotype correlations occurring with this disease. 
S76 The Marfan Syndrome in Neonates Tognato et al.

\section{Funding}

None.

\section{Conflict of Interest \\ None declared.}

\section{References}

1 Amado M, Calado MA, Ferreira R, Lourenço T. Neonatal Marfan syndrome: a successful early multidisciplinary approach. BMJ Case Rep 2014;2014:bcr2013202438

2 Ghandi Y, Zanjani KS, Mazhari-Mousavi SE, Parvaneh N. Neonatal marfan syndrome: report of two cases. Iran J Pediatr 2013;23(01): $113-117$
3 Peng Q Deng Y, Yang Y, Liu H. A novel fibrillin-1 gene missense mutation associated with neonatal Marfan syndrome: a case report and review of the mutation spectrum. BMC Pediatr 2016;16:60

4 Strigl S, Quagebeur JM, Gersony WM. Quadrivalvar replacement in infantile Marfan syndrome. Pediatr Cardiol 2007;28(05):403-405

5 Shih H-Y, Liu W-S, Chen T-J. Neonatal Marfan syndrome - a case report. Acta Cardiol Sin 2004;20:171-175

6 Loeys BL, Dietz HC, Braverman AC, et al. The revised Ghent nosology for the Marfan syndrome. J Med Genet 2010;47(07):476-485

7 Dietz H. Marfan syndrome. In GeneTest: Medical Genetics Information Resource (database online). Copyright, University of Washinton, Seattle. 1997-2011: Available at: http://genetests. org. Accessed April 20, 2011

8 Das R, Majumder B, Bera D, Chakraborty S. Neonatal Marfan syndrome: a rare presentation. Nig J Cardiol 2015;12(01):57-59 